\title{
Gerenciamento dos resíduos eletroeletrônicos dentro da Universidade Federal do
}

\section{Pará}

\author{
Management of electronelectronic waste within the Federal University of Pará \\ Gestión de residuos electronelectrónicos dentro de la Universidad Federal de Pará
}

Recebido: 01/03/2021 | Revisado: 06/03/2021 | Aceito: 12/03/2021 | Publicado: 20/03/2021

\author{
Gabriela Rousi Abdon da Silva \\ ORCID: https://orcid.org/0000-0001-8960-7076 \\ Universidade Federal do Pará, Brasil \\ E-mail: gabrielarousiabdon@gmail.com \\ Jarleson dos Santos Lima \\ ORCID: https://orcid.org/0000-0001-7208-1098 \\ Universidade Federal do Pará, Brasil \\ E-mail: jarleson.lima10@gmail.com \\ Beatriz Xavier de Souza Villaça \\ ORCID: https://orcid.org/0000-0002-0343-5881 \\ Centro Universitário do Pará, Brasil \\ E-mail: jarleson.lima10@gmail.com
}

\begin{abstract}
Resumo
A problemática ambiental relacionada aos resíduos eletroeletrônicos é uma questão bastante discutida, principalmente após a instituição da Política Nacional dos Resíduos Sólidos - Lei n ${ }^{\mathbf{0}}$ 12.305/2010. Os centros de estudos e universidades, considerados polos de avanços tecnológicos, são grandes geradores dessa tipologia de resíduos, assim a implantação de um gerenciamento correto destes eletroeletrônicos torna-se fundamental nesses locais. O presente trabalho busca apresentar a realidade atual do gerenciamento dos resíduos eletroeletrônicos dentro da Universidade Federal do Pará (UFPA), revelando cenários de geração destes, nos anos de 2013, 2014, 2015, 2016 e 2017 , além de mostrar os pontos falhos desse gerenciamento, a modo de apresentar propostas para sua melhora. Foi constatado que a maior produção de resíduos eletroeletrônicos ocorreu no ano de 2014, onde é possível destacar os microcomputadores que são destinados ao Departamento de Almoxarifado e Patrimônio (DAP) da UFPA. Por fim, demonstrou-se que o maior ponto falho no gerenciamento é a falta de comunicação entre os institutos e o DAP, complementado a alta burocracia presente entre os tramites, além da ausência da aplicação da responsabilidade compartilhada. Como pontos de recomendação foram propostos a implantação de ações de educação ambiental com técnicos e servidores e inserção desses resíduos em processos licitatórios de vínculo entre a UFPA e cooperativas que coletem e tratem esse tipo de material.
\end{abstract}

Palavras-chave: Resíduos eletrônicos; Gerenciamento; Geração.

\begin{abstract}
The environmental issue related to electrical and electronic waste is a widely discussed issue, especially after the establishment of the National Policy on Solid Waste- Law No. 12,305 / 2010. The study centers and universities, considered poles of technological advances, are major generators of this type of waste, so the implementation of a correct management of these electronics is essential in these places. This paper seeks to present the current reality of electronic waste management within the Federal University of Pará (UFPA), revealing scenarios of its generation in the years 2013, 2014, 2015, 2016 and 2017, in addition to showing the flaws in this management, in order to present proposals for its improvement. It was found that the largest production of electronic waste occurred in 2014, where it is possible to highlight the microcomputers that are destined to the Department of Warehousing and Heritage (DAP) at UFPA. Finally, it was shown that the biggest flaw in management is the lack of communication between the institutes and the DAP, complemented by the high bureaucracy present between the procedures, in addition to the absence of the application of shared responsibility. As points of recommendation, it was proposed to implement environmental education actions with technicians and civil servants and insert this waste in bidding processes between UFPA and cooperatives that collect and treat this type of material.
\end{abstract}

Keywords: Electronic waste; Management; Generation.

\section{Resumen}

El tema ambiental relacionado con los desechos eléctricos y electrónicos es un tema ampliamente discutido, especialmente después del establecimiento de la Política Nacional de Residuos Sólidos - Ley No. 12.305 / 2010 . Los centros de estudio y las universidades, considerados polos de avances tecnológicos, son grandes generadores de este 
tipo de residuos, por lo que la implementación de una correcta gestión de esta electrónica es fundamental en estos lugares. Este trabajo busca presentar la realidad actual de la gestión de residuos electrónicos dentro de la Universidad Federal de Pará (UFPA), revelando escenarios de su generación en los años 2013, 2014, 2015, 2016 y 2017, además de mostrar las fallas en esta gestión, con el fin de presentar propuestas para su mejora. Se encontró que la mayor producción de residuos electrónicos ocurrió en 2014, donde es posible destacar las microcomputadoras que están destinadas al Departamento de Almacenaje y Patrimonio (DAP) de la UFPA. Finalmente, se demostró que la mayor falla en la gestión es la falta de comunicación entre los institutos y la DAP, complementada por la alta burocracia presente entre los trámites, además de la ausencia de la aplicación de la corresponsabilidad. Como puntos de recomendación, se propuso implementar acciones de educación ambiental con técnicos y funcionarios e insertar estos residuos en los procesos de licitación entre la UFPA y las cooperativas que recolectan y tratan este tipo de material.

Palabras clave: Residuos electrónicos; Gestión; Generación.

\section{Introdução}

Lucena et al. (2016), consideram que a problemática ambiental atrelada a tecnologia não foi uma preocupação inicial, fazendo com que a produção em massa não fosse, originalmente, relacionadas como fontes de poluição ou danosas ao meio ambiente. Nos dias atuais, a tecnologia envolve todas as áreas da sociedade, estando presente na rotina da população, desde escolas, casas e até nos locais de trabalho.

Todos os dias novas tecnologias são descobertas acarretando na corrida para a obtenção de aparelhos mais avançados que acompanhem esse desenvolvimento tecnológico. Bachi, 2013, relata que muitos aparelhos acabam se tornando obsoletos com curtos períodos de vida útil, gerando uma expressiva produção diária de resíduos eletroeletrônicos (REE). A Associação Brasileira de Normas Técnicas (2013) define os eletroeletrônicos como:

“equipamentos, partes e peças cujo funcionamento depende de correntes elétricas ou campos eletromagnéticos, bem como os equipamentos para geração, transmissão, transformação e medição dessas correntes e campos, podendo ser de uso doméstico, industrial, comercial e de serviços”. (ABNT-NBR 16156/2013).

Segundo Baldé et al. (2017) o Brasil é o segundo maior produtor de REE da América como um todo, sendo superado somente pelos Estados Unidos da América. Assim, a realidade brasileira é que o país produziu cerca de 1,5 milhões de toneladas de REE no ano de 2016, onde somente $20 \%$ foi encaminhado para a reciclagem, os autores ainda relatam que até o início de 2017, não haviam políticas específicas no país para esse tipo de resíduos.

Assim, Morais et al. (2020) retrataram que mesmo após 10 anos da promulgação da Política Nacional dos Resíduos Sólidos (Lei nº12.305/2010), uma grande porcentagem da população ainda não tem conhecimento acerca da legislação em questão, onde uma quantidade ainda mais expressiva da população não sabe como deve descartar seu resíduo eletroeletrônico. A realidade relatada acarreta, para alguns, o acúmulo de aparelhos inservíveis no próprio ambiente domiciliar ou descarte indevido junto as outras tipologias de resíduos.

Natume e Anna (2012), explanam que os REE`s tem em sua composição desde elementos simples até hidrocarbonetos complexos, além de grande porcentagem de metal, podendo representar até $70 \%$ de seu conteúdo. Esse é o principal ponto ao estudar os tipos de reciclagem e reutilização dos aparelhos eletrônicos, já que regras gerais não podem ser aplicadas e, cada aparelho deve ser conhecido junto a sua composição e especificidades.

Ressalta-se que entre os materiais simples que os eletroeletrônicos apresentam em sua composição, destacam-se vidros, papeis e plásticos e nem todas as subtipologias desses materiais são passivas de reciclagem, e mesmo os que apresentam essa possibilidade, a maioria não sofre a segregação de maneira correta devido ao desconhecimento dos processos adequados de separação pelas cooperativas (Dullius \& Silva, 2020). Silva Júnior et al. (2020) explanam que além de grande parte da população não ter real noção das consequências danosas que a destinação inadequada dos resíduos eletroetrônicos 
pode causar ao meio ambiente e a saúde humana, uma porcentagem também expressiva não tem conhecimento acerca das possibilidades de reciclagem dessa tipologia de resíduos.

Suemitsu e Araújo, 2015 afirmam que as universidades se destacam como grandes geradores de REE, apresentado tendência ao crescimento de produção desse tipo de resíduo, entretanto, elas, ao mesmo tempo, são grandes fontes de desenvolvimento de tecnologia para auxiliar no gerenciamento, reaproveitamento e reciclagem dessa tipologia de material.

No ano de 2010, entrou em vigor no Brasil a Política Nacional dos Resíduos Sólidos (PNRS) Lei n 12.305/2010, onde é instituída as diretrizes para a gestão integrada e gerenciamento correto dos resíduos sólidos, incluindo os resíduos perigosos, onde partes componentes dos REE se encaixam, além de ressaltar a importância da responsabilidade compartilhada, onde está deve ser aplicada desde o produtor até o consumidor final. Ao passo que, para um gerenciamento correto dos resíduos eletroeletrônicos, é importante que seu quantitativo seja conhecido, assim como as especificações dos subtipos de resíduos, como notebook, impressoras e afins, facilitando o processo de gerenciamento e auxiliando no processo de reciclagem.

Desta forma, o presente trabalho visa apresentar um panorama dos resíduos eletroeletrônicos dentro da Cidade Universitária José da Silveira Netto, realizando comparativo da geração entre os anos de 2014, 2015, 2016 e 2017, além de especificar os subtipos de REE mais produzidos, efetuando discursão acerca do gerenciamento desse tipo de resíduos dentro do campus universitário de Belém, apontando suas falhas e discutindo possíveis melhorias.

\section{Metodologia}

O estudo utilizou o método dedutivo, esse procedimento metodológico visa confirmar a hipótese tomando como base as análises das informações obtidas durante a pesquisa (Pereira et al., 2018). A metodologia utilizada é semelhante a empregada por Watanabe e Candiani (2019), a qual objetivou a investigação minuciosa do processo de gestão de resíduos eletroeletrônicos em instituições de ensino superior.

Deste modo, o presente estudo discute a hipótese do gerenciamento inadequado dos REE's na Universidade Federal do Pará (UFPA) e para confirma-la a investigação foi dividida em quatro etapas. A primeira etapa constituiu-se na pesquisa bibliográfica acerca dos REE no contexto mundial, nacional e especificamente nas universidades, além de questões relacionadas a seu gerenciamento, reciclagem e reutilização. Nesta etapa realizou-se a leitura e análise de decretos, leis e resoluções de esfera federal e estadual.

$\mathrm{Na}$ etapa posterior ocorreu o levantamento dos dados e a trabalhabilidade sobre eles, realizando análises estatísticas básicas. A terceira etapa se ateve a simples observação no campus Belém, a fim de averiguar e constatar visualmente a disposição inadequada desses resíduos. A quarta, e última, etapa foi a discussão acima de tudo que foi observado e sobre o tipo de gerenciamento que está sendo empregado no campus universitário.

\section{Resultados e Discussão}

Faria et al. (2015) apresentou como é feito o gerenciamento dos REE dentro da Cidade Universitária José da Silveira Netto (Figura 1). Resumidamente, o REE é misturado aos outros patrimônios considerados inservíveis (mesas, cadeiras, entre outros), segundo os institutos, onde são alocados em lugares inapropriados à espera da coleta a ser realizada pela Diretoria de Almoxarifado e Patrimônio (DAP), esta fica responsável pelo destombamento dos materiais e posterior destinação final, onde os materiais são disponibilizados para outros institutos ou para doações externas. 
Figura 1 - Fluxograma de etapas simplificadas do gerenciamento de REE dentro da UFPA.

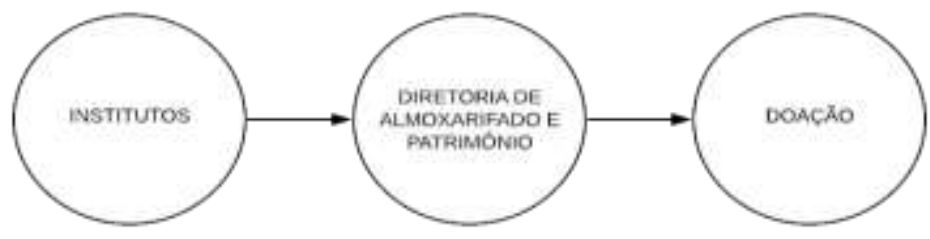

Fonte: Adaptado de Faria et al. (2015).

Dentro do atual processo de gerenciamento dos REE empregado na UFPA, é importante ressaltar que os materiais são requeridos por professores, os quais encaminham essa necessidade aos institutos, que por sua vez requerem a reitoria. Entretanto, cada material requerido fica correlato ao nome do professor solicitante ou ao projeto/laboratório vinculado e não em nome do instituto responsável.

O estudo de Faria et al. (2015) relataram que o DAP, por ser responsável por toda a Universidade Federal do Pará (UFPA), não consegue vencer a grande demanda de serviço, ocasionando o acondicionamento irregular dos materiais em vários pontos da UFPA (Figura 2).

Figura 2 - Acondicionamento inadequado de materiais considerados inservíveis dento da UFPA.
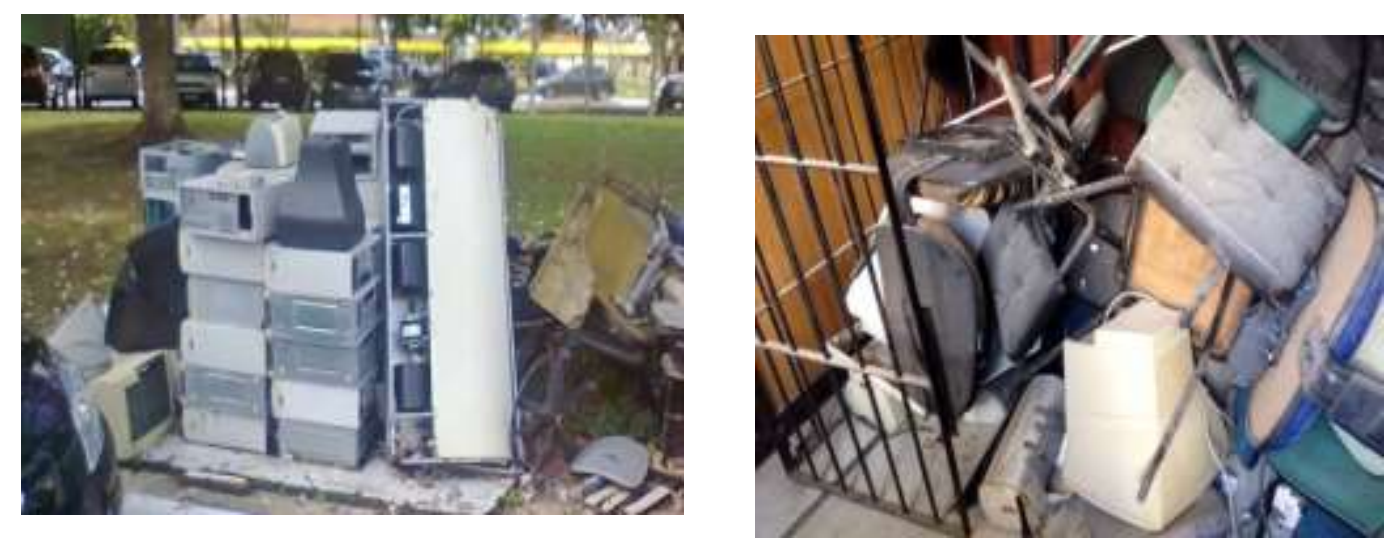

Fonte: Faria et al. (2015).

Nos dias atuais a disposição irregular já não é tão notória dentro da Universidade, entretanto, fatos, como a disposição de componente de computadores as margens do rio Tucunduba (Figura 3) que corta a universidade, ainda são observados, essa disposição pode acarretar lixiviação dos metais tanto para o solo como para a água do rio. 
Figura 3 - Disposição inadequada de resíduos eletroeletrônicos as margens do rio Tucunduba.

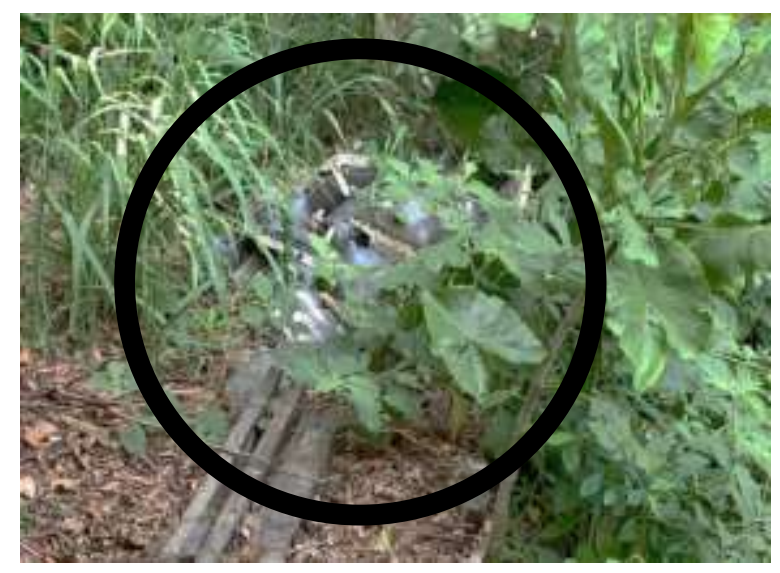

Fonte: Autores (2019).

Faria et al. (2015), apresentaram um levantamento dos REE produzidos na UFPA em 2013, para o campus Belém. Assim foi possível realizar um comparativo da produção de 2013 até 2017. O gráfico que apresenta o quantitativo de REE produzido em cada um dos anos de estudos é apresentado na Figura 4.

Figura 4 - Quantitativo de REE “destombados” por ano na UFPA- campus Belém.

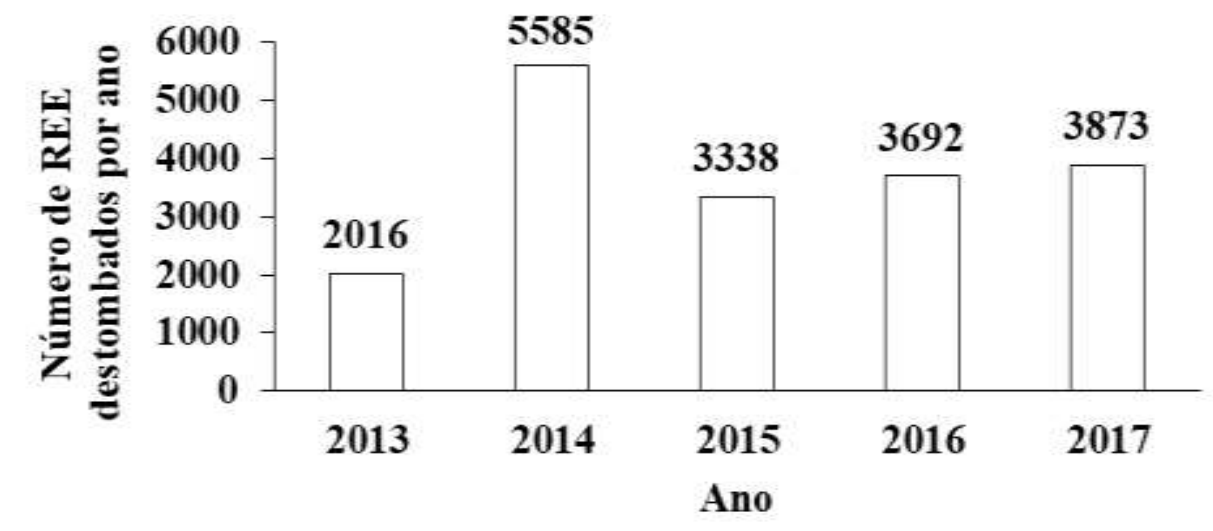

Fonte: Autores (2019).

A partir dos dados é possível perceber um crescimento na produção de resíduos eletrônicos na universidade, onde a maior porcentagem de crescimento foi observada entre 2013 e 2014 . O crescimento menos acentuado observado nos outros anos é explicado pela durabilidade dos materiais eletroeletrônicos.

Um fator importante de se ressaltar é que ainda são coletados nos institutos aparelhos obsoletos como retroprojetor (Figura 5), máquina de escrever, entre outros, isso é explicado por dois pontos, o primeiro é que alguns aparelhos requeridos chegam após longos períodos desde sua solicitação, fazendo com que uma pequena parcela destes não sejam tão atuais na hora de sua entrega, como esperava-se quando foram solicitados, outro ponto é a grande burocracia para que o destombamento do material seja realizado, fazendo com que, muitas vezes, os institutos prefiram alocar os materiais em lugares inadequados e acabam por não contatar o DAP para realizar o destombamento, o que produz como consequência várias formas de poluição, entre elas a visual. 
Figura 5 - Retroprojetor nunca utilizado.

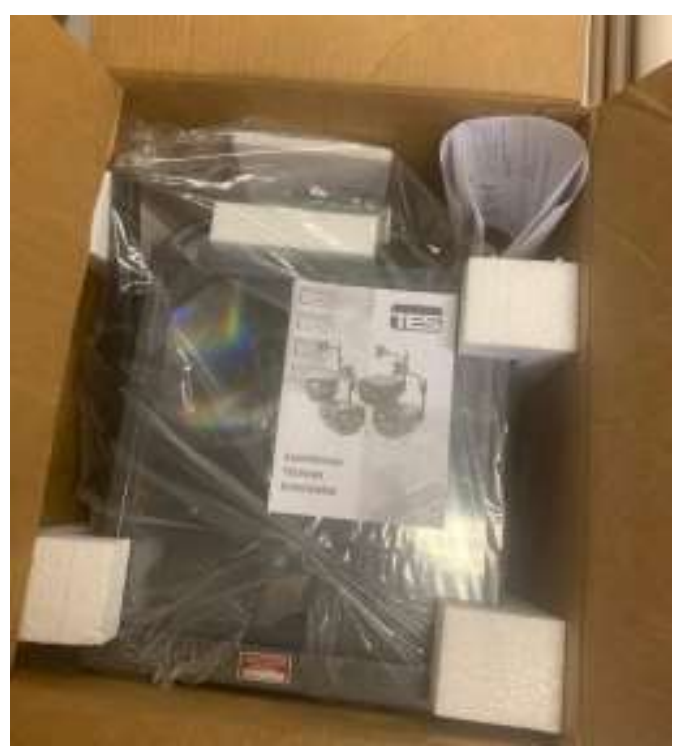

Fonte: Autores (2019).

Dentre os resíduos eletroeletrônicos gerados, o que apresenta maior quantidade são os microcomputadores, onde estes representam em média 19,8\% de todo o REE gerado na universidade. O ano de 2016, foi o ano que presentou menor porcentagem de microcomputadores destinados ao DAP, neste ano a maior quantidade de REE gerado foi referente aos "No Break".

O ano de maior produção de resíduos eletrônicos dento da UFPA, foi o ano de 2014, onde foram produzidos 5585 resíduos eletroeletrônicos, um crescimento de aproximadamente 177\% em relação ao ano anterior. Em 2014 os REE mais gerados na universidade foram: microcomputadores, condicionador de ar, telefone e impressoras, representando 16,11\%, $11,30 \%, 10,78 \%$ e 9,58\%, respectivamente, do total gerado. Entre os anos de 2014 e 2015 ocorreu grande queda na geração desses resíduos, entretanto, a partir de 2015 o crescimento voltou a ocorrer, de forma menos acentuada, porém ainda sim significativa.

Considerando a classificação apresentada pela Associação Brasileira de Desenvolvimento Industrial (ABDI), em seu estudo denominado "Logística Reversa e Equipamentos Eletroeletrônicos" no ano de 2013, os resíduos eletrônicos gerados na UFPA, foram divididos em Linha Marrom, Linha Branca, Linha Verde e Linha azul, sendo acrescida da Linha Amarela fazendo referência aos resíduos que não se encaixam em nenhum dos grupos apresentados pela ABDI.

Assim, foram gerados gráficos referentes aos percentuais de produção de resíduos eletrônicos segundo sua linha de classificação, todos apresentados na Figura 6. Onde é possível analisar com precisão que a maior porcentagem de resíduos eletroeletrônicos gerados nos últimos anos pertence a Linha Amarela. 
Figura 6 - Percentual de resíduos gerados segundo sua linhagem.

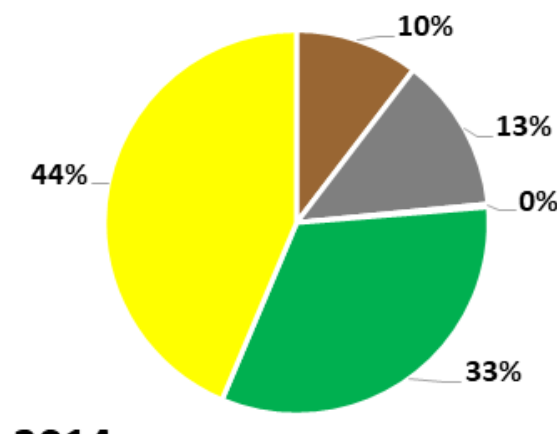

2014

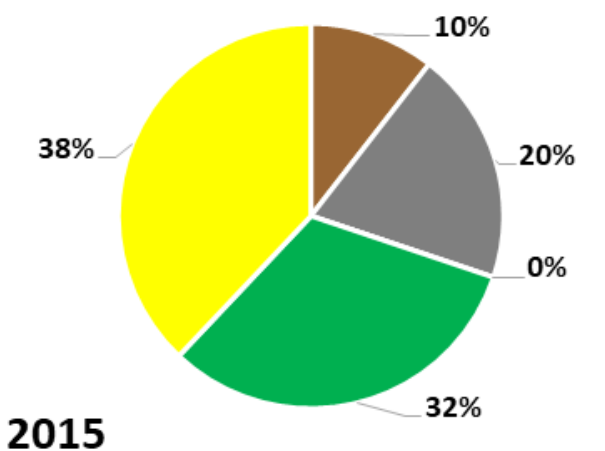

2015
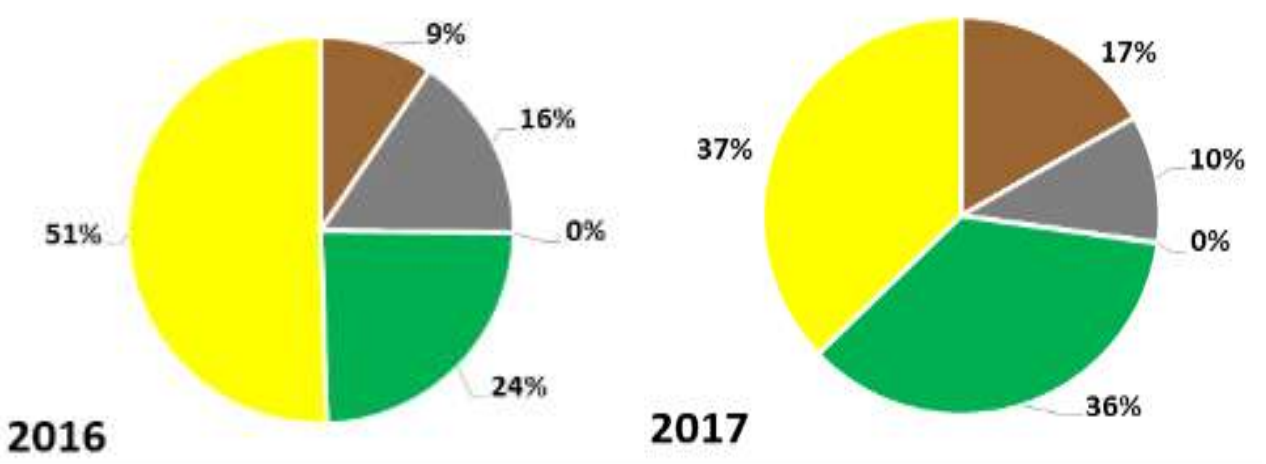

Onde: Marrom=Linha Marrom; Amarelo = Linha Amarela; Verde= Linha Verde; Branco= Linha Branca. Fonte: Autores (2019).

A Linha Amarela é seguida pela expressiva porcentagem de eletroeletrônicos pertencentes a Linha Verde, a qual é representada segundo a ABDI, como uma linha composta por materiais com período de duração intercalando entre 2 e 5 anos, com peso variando entre $0,09 \mathrm{Kg}$ até $30 \mathrm{Kg}$, e sendo composto principalmente por metais pesados e plástico. Os eletroeletrônicos referentes a Linha Branca ocuparam $3^{\circ}$ lugar durante os anos 2014, 2015 e 2016, sendo superado pelos da Linha Marrom somente no ano de 2017. Esse fato pode ser explicado, já que a Linha Branca é composta principalmente por Refrigeradores e Compressores de ar, e ambos são amplamente utilizados, tanto em sala de aula como em laboratório de pesquisas dentro da Universidade, esses tipos de materiais já se apresentam como materiais de grande porte chegando até 70 $\mathrm{Kg}$, tendo vida útil longa e sendo compostos principalmente por metais.

Os equipamentos da Linha Azul se apresentaram com quantidade insignificante de produção, desta forma são os de menor importância, mas não esquecíveis nesse estudo, estes são equipamentos de pequeno porte com vida útil de longa duração e compostos principalmente por vidro e metais, podendo ser exemplificado por liquidificadores.

A maior porcentagem apresentada como outros é explicada haja vista que foram destombados muitos instrumentos de pesquisas laboratoriais que não são de uso comum, além de peças segregadas de produtos, que não podem ser generalizadas, não se encaixando assim em nenhuma das outras linhas.

Dentro da UFPA, funciona efetivamente a coleta seletiva, como relatou, Almeida, (2011), onde materiais como papel, plástico, metal, são destinados a cooperativas, entretanto os resíduos eletroeletrônicos não estão inclusos nessa coleta, devido à dificuldade e periculosidade de reciclagem e reutilização desses resíduos e a burocracia para o destombamento desses materiais. Faria et al. (2015) demonstram pontos falhos dentro do gerenciamento de resíduos inservíveis como um todo, onde o pior ponto é a inexistências ou atraso das declarações que devem ser feitas pelos institutos para que o DAP possa recolher o material. 
A Política Nacional dos Resíduos Sólidos (PNRS), Lei no 12.305/2010, apresenta como um de seus instrumentos a logística reversa, onde é encaminhado ao produtor o resíduo, para que ele possa reinserir no ciclo produtivo ou destinar corretamente esse resíduo. Nascimento et al. (2018) enfatizam que a prática da logística reversa é o ponto principal para a realização correta do processo de descarte dos materiais eletroeletrônicos, mesmo que esse descarte ocorra em alguns dias ou alguns anos após o uso. Essa dinâmica não é realizada dentro da UFPA, o que é consequência da presença de vários obstáculos. Neste contexto, Bernardo et al. (2020) explicam que a dificuldade do trânsito de informações entre fabricantes e os locais de desmontagem é o que mais complexifica a aplicação do sistema de fluxo reverso dos materiais. Outro ponto a ser elencado é responsabilidade compartilhada, outro instrumento da PRNS, o qual também não é efetivo, realidade confirmada por Faria et al. (2015).

A dificuldade da efetividade da logística reversa é, em grande parte, da dimensão da universidade, além da variedade de produtores desses resíduos eletroeletrônicos que circulam na UFPA, assim o contato e segregação por marca é um trabalho dispendioso e efetivo. Um ponto de melhora nesse quesito seria a realização de convênio entre a universidades e as grandes marcas de produtores desses materiais, principalmente computadores e microcomputadores, podendo não só facilitar a questão da logística reversa como também auxiliar na facilitação de alunos e técnicos para obtenção de aparelhos da marca em questão.

Outra ação que pode ser executada é a cooperação entre cooperativas que realizem a desmontagem e/ou reciclagem dos REE's. Adriato e Catilho (2020) evidenciam a importância das cooperativas na diminuição das consequências negativas relacionadas a essa tipologia de resíduos, como a diminuição do consumo de matérias primas até então invioladas e a minimização das tipologias de poluições ambientais associadas.

Quanto a burocracia para o processo de destombamento, seria interessante que os eletroeletrônicos fossem requeridos e depois destombados em nome dos institutos ou das faculdades e não em nome de um professor responsável, repassando a responsabilidade pelo acionamento do DAP as secretarias dos institutos. Assim, situações, tais como a de eletroeletrônicos requeridos por professores já aposentados não existiriam, o que diminuiria o número de aparelhos obsoletos alocados pelo campus.

Além da burocracia para seu destombamento, as doações desses materiais é outro ponto a ser discutido, já que o DAP disponibiliza a doação para colégios e projetos filantrópicos, apenas repassando o problema, não efetivando a responsabilidade compartilhada, assim esse ponto pode ser trabalhado com a inserção desses materiais eletroeletrônicos a um tipo de licitação como é feita para os outros resíduos recicláveis, onde cooperativas aptas recolhessem esses resíduos e realizassem a correta segregação de suas partes e posterior destinação final, desta forma, com processo licitatório a UFPA teria evidências e documentos que comprovem que os resíduos estarão sofrendo o tratamento correto.

Outro tópico benéfico, relacionado ao processo licitatório, seria o de cunho socioeconômico para as cooperativas e ambiental para o planeta, já que estes materiais poderiam ser novamente utilizados como matéria prima na fabricação de novos produtos eletroeletrônicos, assim caracterizando a recirculação proposta pela economia circular como enfatiza Xavier e Lins (2018).

De maneira resumida, uma possibilidade de gerenciamento simplificado desses resíduos é apresentada na figura 7 , onde há a inserção mais adequada dos institutos e das cooperativas. 
Research, Society and Development, v. 10, n. 3, e38610313456, 2021

(CC BY 4.0) | ISSN 2525-3409 | DOI: http://dx.doi.org/10.33448/rsd-v10i3.13456

Figura 7 - Proposta de gerenciamento simplificado dos resíduos eletroeletrônicos dentro da UFPA.

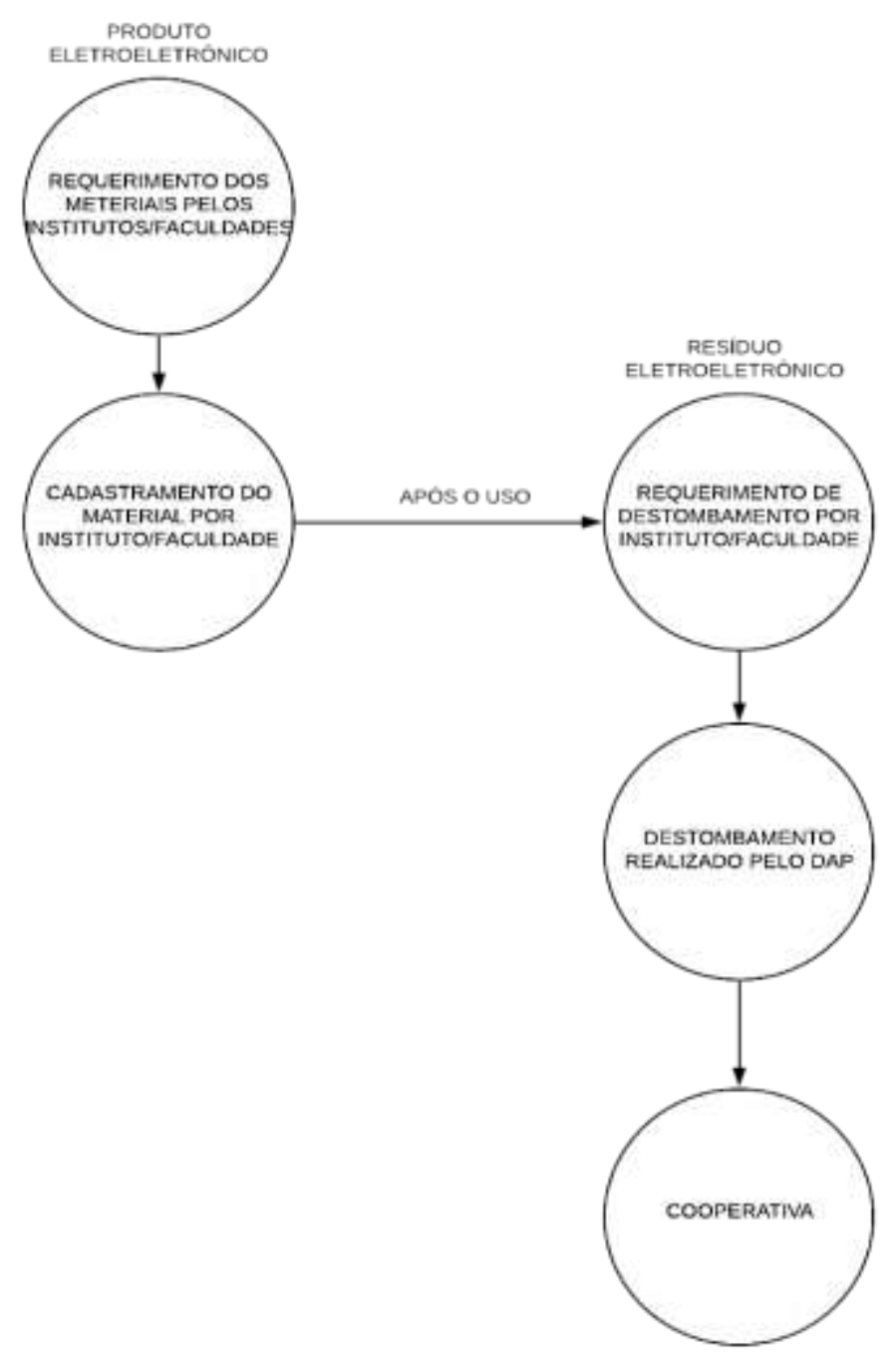

Fonte: Autores (2019).

Evidencia-se que a aplicabilidade e eficácia da gestão adequada dessa tipologia de resíduos só é possível a partir de uma abordagem multidisciplinar onde, todos os envolvidos no processo de gestão, estejam cientes de seus respectivos papeis dentro do sistema para que o fluxo siga de maneira satisfatória (Cardoso et al., 2019).

As cooperativas em Belém que recebem REE’s estão apresentadas na Tabela 1. Essas cooperativas podem ser convocadas pela Universidade Federal do Pará (UFPA), para a avaliação do interesse no processo licitatório. 
Tabela 1 - Cooperativas e ONG's que recebem REE's em Belém.

\begin{tabular}{ll}
\hline COOPERATIVAS/ ONG's & SITE \\
\hline CONCAVES & http://concaves.blogspot.com/ \\
DESCARTE JÁ & https://lixoeletronicobelem.wordpress.com/ \\
NO OLHAR & http://www.noolhar.org.br/ \\
ACCSB & http://cempre.org.br/servico/cad-det/6036/accsb---assoc--catad--da-coleta-seletiva-de-belem \\
ARAL & https://www.facebook.com/catadoresdaaral/ \\
\hline
\end{tabular}

Fonte: Operador Verde Senac (2016).

Nota-se que, em Belém, ainda são poucas as cooperativas que recebem os REE's, essa realidade é constatada nas demais regiões brasileiras, diferenciando-se somente as regiões sul e sudeste que detém a maior quantidade de cooperativas, empresas e ONG's que atuam no mercado de reciclagem e/ou reutilização desses tipos de resíduos (Araujo \& Xavier, 2019). Esse fato é um dos pontos que dificultam a aplicabilidade de um gerenciamento eficaz desse tipo de material.

\section{Conclusão}

De acordo com o exposto, é evidente que o gerenciamento dos resíduos eletroeletrônicos dentro da Universidade Federal do Pará é um ponto que deve ser estudado e estruturado, já que a geração desse tipo de resíduo é expressiva.

Assim algumas atitudes devem ser tomadas, destacando a necessidade da implementação de um sistema onde o destombamento de materiais inservíveis seja realizado de forma mais rápida. Entende-se que por ser uma instituição pública deve-se ter o total cuidado com os aparelhos que são adquiridos e posteriormente são doados/destinados, entretanto o modo que se apresenta atualmente, o processo de gerenciamento dos resíduos eletroeletrônicos dentro da UFPA está sendo ineficaz e em desacordo com o que frisa a PNRS.

Destaca-se que o processo de licitação com cooperativas é uma forma de implementar a responsabilidade compartilhada dentro da UFPA, já que não apenas repassaria a responsabilidade e sim controlaria qual tipo de reciclagem e/ou reutilização a cooperativa fará com os resíduos, por meio de documentação.

Outro ponto a ser destacado dentro do contexto de gerenciamento de resíduos eletroeletrônicos é o controle, a ser realizado pelo DAP por meio de sistema informacional, da geração de resíduos por parte dos institutos, já que esse tipo de acompanhamento e físcalização possui tendência a menor geração e disposição inadequadas desses resíduos pelos institutos.

Reitera-se que ainda são poucas as empresas, $O \mathrm{NG}^{\prime}$ 's e cooperativas que aceitam esse tipo de material, tanto na região de estudo quanto ademais, tornando assim crucial que o governo atue juntamente a este setor, afim de promover meios de aperfeiçoamento das cooperativas já existentes e a viabilização da criação de novas cooperativas que trabalhem de maneira especializada com os REE's.

É importante que algumas ações de educação ambiental sejam realizadas com os responsáveis técnicos, secretariados dos institutos, discentes e docentes de todas as áreas e todos os envolvidos no processo de gestão, para que sejam explanadas as consequências da disposição inadequada e o má gerenciamento desses resíduos, além de enfatizar a responsabilidade compartilhada entre todos os setores, para que todos tenham consciência de seus atos e suas possíveis consequências.

Por fim, aconselha-se que sejam realizados levantamentos anuais e a criação de um banco de dados efetivo de cunho qualiquantitativo para controle dos REE’s dentro da Universidade Federal do Pará. É de extrema importância que outros estudos sejam realizados com finalidade de automatizar e facilitar os cursos burocráticos durante o processo de gerenciamento desses materiais considerados inservíveis. 


\section{Referências}

ABNT NBR 16156, de 18 de abril de 2013. Resíduos de equipamentos eletroeletrônicos - Requisitos para atividade de manufatura reversa: Associação Brasileira de Normas Técnicas.

Andriato, M. F., \& Castilho, M. L. (2020). Análise da sustentabilidade de cooperativas de materiais recicláveis selecionadas: alternativas para o tratamento de resíduos sólidos urbanos. Acta Scientiarum. Human and Social Sciences, 42(1), e51002-e51002.

Almeida, L. D. F. (2011). A gestão de resíduos sólidos em contextos intraorganizacionais: um estudo a partir da UFPA. Dissertação de mestrado. Universidade Federal do Pará, Núcleo de Altos Estudos Amazônicos, 155p.

Araujo, R. A. de., \& Xavier, L. H. da S. M. (2019, julho). Banco de dados e estudo geoespacial de organizações do segmento de resíduos eletroe letrônicos no Brasil. XXVII Jornada de Iniciação Científica e III Jornada de Iniciação em Desenvolvimento Tecnológico e Inovação, Rio de Janeiro, RJ, Brasil. <http://mineralis.cetem.gov.br/bitstream/cetem/2277/1/Ra\%C3\%ADssa\%20Andr\%C3\%A9\%20de\%20Araujo.pdf> Acesso em: 19 de janeiro de 2021.

Bachi, M. H. (2013). Resíduos Tecnológicos: A relação dos Resíduos Eletroeletrônicos e a Legislação no Brasil. Revista Brasileira de Gestão Ambiental, 7(1), $01-05$.

Baldé, C. P., Forti, V., Gray, V., Kuehr, R., \& Stegmann, P. The Global E-waste Monitor - 2017, United Nations University (UNU), International Telecommunication Union (ITU) \& International Solid Waste Association. (ISWA), Bonn/Geneva/Vienna.

Bernardo, O. O., Souza, M. T. S. de., \& Demajorovic, J. (2020). Inovação na cadeia reversa de resíduos eletroeletrônicos: Um estudo sobre os sistemas de informação e as tecnologias de rastreamento. Revista de Administração de Empresas, 60(4), 248-261.

Cardoso, E., Rotolo, M., Valle, T. F., Ottoni, M. de S. O., \& Fernandes, H. F. (2019). Desafios da Logística Reversa de Equipamentos Eletroeletrônicos no Brasil. Revista Ineana, 7(1), 6-19.

Dullius, A., \& Silva, M. C. da. (2020). Dinâmicas territoriais dos resíduos eletrônicos domiciliares na cidade de Matinhos-PR. Estudo \& Debate, 27(2), 149169. https://doi.org/10.22410/issn.1983-036X.v27i2a2020.2547

Faria, A. S. D., Ferreira, C. E. C., Meireles, J. L. de S., Silva, G. R. A. da., \& Braga, R. M. Q. L (2015, outubro). Um panorama do gerenciamento dos resíduos eletrônicos na Cidade Universitária José da Silveira Netto - UFPA/PA. Congresso Brasileiro de Engenharia Sanitária e Ambiental, Rio de Janeiro, RJ, Brasil, 28 .

Giannetti, B. F., Bonilla, S. H., \& Almeida, C. M. V. B. (2012). Cleaner production initiatives and challenges for a sustainable world. Journal of Cleaner Production, 22(1), I. https://doi.org/10.1016/S0959-6526(11)00431-8

Lei 12.305, de 2 de agosto de 2010. Institui a Política Nacional de Resíduos Sólidos; altera a Lei n 9.605 , de 12 de fevereiro de 1998 e dá outras outras providências. Brasília, DF. http://www.planalto.gov.br/ccivil_03/_ato2007-2010/2010/lei/112305.htm.

Lucena, L. B. de., Soares, E. de F. A., Batista, L. M. de S. D., Pereira, T. de O., \& Gomes, A. F. (2016). Reltec: uma proposta para o problema dos resíduos tecnológicos local. Revista Extendere, 4(2), 45-53. http://periodicos.uern.br/index.php/extendere/article/view/2331

Morais, M. de. O., Brejão, A. S., Silva, U. J. da., \& Neto, J. S. (2020). Dez anos da política nacional de resíduos sólidos: um estudo comparativo entre 2011 e 2020 sobre o entendimento dos consumidores referente ao descarte de equipamentos eletroeletrônicos. Brazilian Journal of Development, 6(11), 91851-91873. Natume, R. Y., \& Sant' Anna, F. S. P. (2011, maio). Resíduos eletroeletrônicos: um desafio para o desenvolvimento sustentável e a nova Lei da Política Nacional de Resíduos Sólidos. Internacional Workshop on Advances in Cleaner Production, São Paulo, Brasil, 3.

Nascimento, F. B., Silva, Y. B. R. da., Lima, L. S. da S., \& Santos, M. D. S. F dos. (2018). Logística reversa dos resíduos de equipamentos eletroeletrônicos de pós-consumo na cidade de Teresina. Sistemas \& Gestão, 13(4), 519-531.

Pereira, A. S., Shitsuka, D. M., Parreira, F. J., \& Shitsuka, R. (2018). Metodologia da pesquisa científica. UFSM. https://repositorio.ufsm.br/bitstream/handle/1/15824/Lic_Computacao_Metodologia-Pesquisa-Cientifica.pdf?sequence=1 .

Reis, I. (2016). Pontos de coleta e Organizações que trabalham com coleta de resíduos tecnológicos. https://operadorverdesenac.wordpress.com/2016/07/27/pontos-de-coleta-e-organizacoes-que-trabalham-com-coleta-de-lixo-tecnologico/.

Silva Júnior, V. M. da., Dias, G. F. de M., Costa, R. A. da., Miranda, S. B. D. de A., \& Lima, D. R. N. (2020). Percepção sobre o lixo eletrônico: estudo de caso em uma Instituição Federal de Ensino. Research, Society and Development, 9(11), e82091110550-e82091110550.

Suemitsu, W. I., \& Araújo, M. G. (2015). Gestão de resíduos eletroeletrônicos no Centro de Tecnologia da UFRJ. Revista de Sustentabilidade e Tecnologia Ambiental, 10(1), 60-65.

Watanabe, F. P., \& Candiani, G. (2019). Gestão de resíduos de equipamentos eletroeletrônicos em instituições de ensino superior. Revista Ibero-Americana de Ciências Ambientais, 10(5), 169-186.

Xavier, L. H., \& Lins, F. A. F. (2018). Mineração Urbana de resíduos eletroeletrônicos: uma nova fronteira a explorar no Brasil. Brasil Mineral, 379(1), 22-26. 